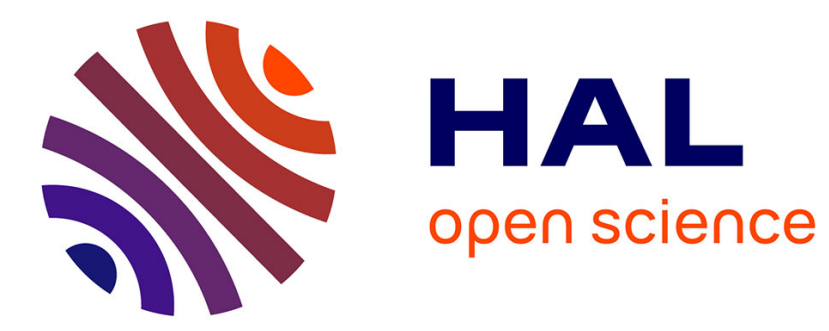

\title{
Simulation and fabrication of silicon nitride microring resonator by DUV lithography
}

Giuseppe A Cirino, Luis A Barea, Antonio A von Zuben, Hervé Lhermite, Bruno Bêche, Olivier de Sagazan, Newton Frateschi, Tayeb M-Brahim

\section{To cite this version:}

Giuseppe A Cirino, Luis A Barea, Antonio A von Zuben, Hervé Lhermite, Bruno Bêche, et al.. Simulation and fabrication of silicon nitride microring resonator by DUV lithography. IEEE conference publications, 2016, pp.1 - 4. 10.1109/SBMicro.2016.7731346 . hal-01398394

\section{HAL Id: hal-01398394 \\ https://hal.science/hal-01398394}

Submitted on 17 Nov 2016

HAL is a multi-disciplinary open access archive for the deposit and dissemination of scientific research documents, whether they are published or not. The documents may come from teaching and research institutions in France or abroad, or from public or private research centers.
L'archive ouverte pluridisciplinaire HAL, est destinée au dépôt et à la diffusion de documents scientifiques de niveau recherche, publiés ou non, émanant des établissements d'enseignement et de recherche français ou étrangers, des laboratoires publics ou privés. 


\title{
Simulation and Fabrication of Silicon
}

\section{Nitride Microring Resonator by DUV Lithography}

\author{
Giuseppe A. Cirino ${ }^{1,2}$, Luis A. Barea ${ }^{1}$, Antonio A. von Zuben ${ }^{4}$, Hervé L’hermite ${ }^{2}$, Bruno Beche ${ }^{3}$, \\ Olivier De Sagazan ${ }^{2}$, Newton Frateschi ${ }^{4}$ and Tayeb M-Brahim ${ }^{2}$ \\ ${ }^{1}$ Departamento de Engenharia Elétrica - Universidade Federal de São Carlos - São Paulo, Brazil \\ ${ }^{2}$ IETR - Université Rennes1 - Rennes, France \\ ${ }^{3}$ IPR - Université Rennes1 - Rennes, France \\ ${ }^{4}$ IFGW - Universidade Estadual de Campinas - São Paulo, Brazil
}

e-mail address of corresponding author: gcirino@ufscar.fr

\begin{abstract}
This work reports the design and fabrication of silicon nitride-based microresonators by employing DUV optical lithography and ICP-RIE plasma etching. Microring devices with high $Q$ factors provide high sensitivity and low detection limit, enabling their use in biochemical sensing applications. With these properties, the devices can be used to detect and quantify the biomolecules present in a homogeneous solution, by detecting an effective refractive index change, without using fluorescent labels.
\end{abstract}

Keywords - Microcavity resonator; Silicon photonics; DUV Lithography; Evanescent coupling; Biochemical sensing.

\section{INTRODUCTION}

Nowadays sensing physical quantities by using miniaturized sensors represents a very active area of research. A particular demand exists for the detection of biochemical species. Several optical techniques have proven to be quite effective - leading to very high sensitivity - particularly optical biosensors based on resonant cavities. Micro-sized resonators (MR) play an important role in the success of silicon photonics, because silicon enables resonators of an unprecedented small size. Such devices have different geometries with different confining principles and unique spectral properties, including narrow line-width, high stability, and tunability [1].

Typical optical waveguide sensors utilize evanescent wave to probe the presence of analytes at the sensor surface or in the surrounding medium by detecting the effective index change. Such a sensing scheme is able to eliminate the need of fluorescent labeling of the analyte molecules. In order to detect low concentrations or minute amounts of analytes, a long interaction length is often required for a detectable phase shift. In the early 2000 , microresonators have been proposed in sensing applications [2-4]. Since than, optical microresonators are extensively described in literature [5-7].
High quality factor $(Q)$ and low detection limit in compact devices are the most important features for sensing applications, where the change in $Q$ or resonant wavelength can be used for measuring the change in ambient parameters in the surrounding environment or binding phenomena on the resonator surface. Optical modes are confined in the microcavity by total internal reflections (TIR) at the waveguide interface. When a micro or nanoscopic object like a bacterium or a molecule is brought in contact with the confined circulating light, the interaction will produce a shift in the resonant wavelengths. This can be done by chemically modifying the ring surface, to respond to e.g. bacteria and virus presence and specific gases for environment monitoring $[8,9]$. Among the existing biochemical sensors, planar optical sensors are promising due to their robustness, easy patterning of reagents, and simple incorporation of various materials such as polymers, metals, and dielectrics [1]. In addition, they have high integration capability with electronic devices as well as photonic devices, and can be easily fabricated with mature semiconductor processing technologies, enabling compatibility with microfluidics and MEMS [10].

This work reports the design and fabrication of a low-cost ring MR, closely coupled with two straight bus waveguides, which serve as optical input and output for the device. In order to obtain a $470 \mathrm{~nm}$ gap in the coupling region, a simple contact DUV optical lithography at $248 \mathrm{~nm}$ wavelength was performed.

\section{SPECTRAL PROPERTIES OF MICRORESONATORS}

A generic ring resonator consists of a ring waveguide as a resonant cavity, which is closely coupled with one or two straight bus waveguides, which serve as optical input and output for the device. The characteristics of microring device having finite loss are shown in figure 1a. The characteristic parameters of a typical spectral response are indicated in figure $1 \mathrm{~b}$. The resonances occur when the round-trip phase acquired by the guided wave is equal to multiples of $2 \pi$. The 
resonant wavelengths can then be determined by

$$
\lambda_{\text {RES }}=\frac{n_{E F F} L}{m}, \quad(m=1,2,3 \ldots)
$$

where $\lambda_{R E S}$ is the resonant wavelength, $n_{E F F}$ is the effective index of the guided mode and $L$ is the circumference of the microring.

The spacing between these resonances, called free spectral range $(F S R)$, depends on the resonator length as

$$
F S R=\frac{\lambda^{2}}{n_{G} L}
$$

where $\lambda$ is the free space wavelength, $n_{G}$ is the group velocity and $L$ is the round trip length.

For many applications it is preferred to have a relatively large FSR (several $\mathrm{nm}$ ), implying the use of small rings. This translates into a very hard requirement for the optical waveguide: to make a compact ring, a small bend radius is required, and this is only possible with high-contrast waveguides with strong confinement. A ring resonator as a stand-alone device only becomes useful when there is a coupling to the outside world. The most common coupling mechanism is using co-directional evanescent coupling between the ring and an adjacent bus waveguide. The transmission spectrum of the bus waveguide with a single ring resonator shows a significant drop in the light intensity around the ring resonances. In this sense, the ring resonator behaves as optical filter, and can be used for sensing: the position of the resonance dips is very sensitive to a variety of environmental effects [12].

The sensitivity of a microring sensor is determined by the $Q$ factor of the microresonator. Small change in the effective index $(\delta n E F F)$ can be detected by measuring the resonance shift $\delta \lambda_{R E S}$.

According to the resonance condition of equation 2 , one can write $\left(\delta n_{E F F} / n_{E F F}\right)=\left(\delta \lambda_{R E S} / \lambda_{R E S}\right) \propto(1 / Q)$. The on-off behavior of the pass-port (fig. 1) is defined as extinction ratio $(E R)$ and it is given by

$$
E R=\frac{(r+a)^{2}(r-a)^{2}}{(1+r a)^{2}(1-r a)^{2}}
$$

where $a$ is the single-pass amplitude transmission, and $r$ is the self-coupling coefficient. The finesse is defined as the ratio of FSR and resonance width, and is a measure of the sharpness of resonances relative to their spacing.

$Q$ is a measure of the sharpness of the resonance relative to its central frequency.
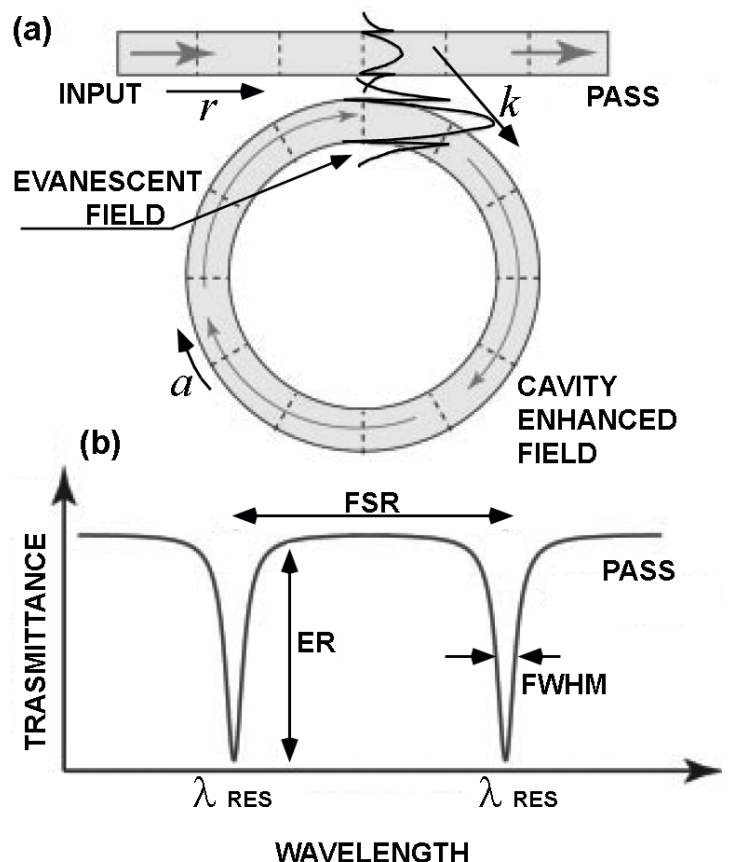

Figure 1: (a) Schematic of a waveguide-coupled microring resonator. Arrows indicate the traveling-wave propagation directions; (b) Schematic of the throughput transmission spectrum showing the single-mode microring resonances, adapted from reference [15].

The physical meaning of the finesse and $Q$ relates to the number of round-trips made by the energy in the resonator before being lost to internal loss and the bus waveguides.

\section{Simulations Results}

The schematic view of the proposed device is illustrated in fig. 2 .
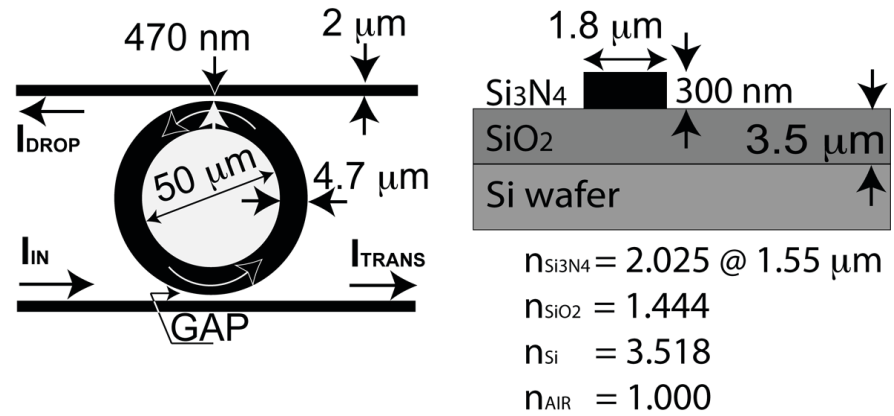

Figure 2: Schematic (a) top and (b) cross section views of the device layout with key features.

The device was simulated by using RSoft Photonic Device Software. The optical modes were calculated using the BeamPROP module, based on the Beam Propagation Method (BPM), for the cross section waveguide showed in Fig. $2 b$. The electric field distributions for the fundamental modes on the $\mathrm{x}$ and $\mathrm{y}$ direction, supported by the waveguide at $1.55 \mu \mathrm{m}$ wavelength, are shown in Fig. 3(a) and 3(b), respectively. 


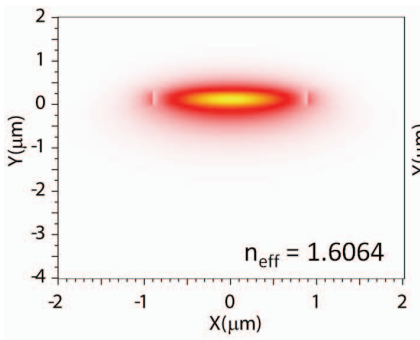

(a)

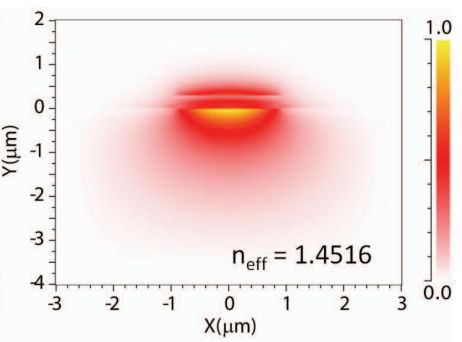

(b)
Figure 3: Fundamental optical modes calculated for the $\mathrm{Si}_{3} \mathrm{~N}_{4}$ waveguide at $1.55 \mu \mathrm{m}$ wavelength with their respective effective refractive indices for (a) TE-polarized light and (b) TM-polarized light.

The spectral response of the resonant microcavity device, was simulated by 2D Finite-Difference Time-Domain (FDTD) method, employing the FullWAVE module from RSoft. The grid size used was $50 \mathrm{~nm}$. Perfectly Matched Layers (PML) method was applied as spatial boundaries around the device, acting as absorption layers of electromagnetic waves. The electromagnetic field that was launched in the input port of the waveguide consists of a delta function in time domain, which allows excitation within large wavelength range.

The spatial distribution of this pulse was Gaussian, centered at $1.55 \mu \mathrm{m}$. A Fast Fourier Transform (FFT) was performed in the time evolution of the field component, allowing obtain the device's resonances, as shown in Fig. 4. It is well known that in the core/cladding interface, the normal component of displacement $D=\varepsilon E$ must be continuous. Thus, the field amplitude at the cladding side should be stronger for a mode with the dominant E-field polarized normal to this interface. For sensor applications, it is important that this discontinuity occurs on the top surface, increasing the sensor detection ability due to longer range of the evanescent field. For this reason, only the TM polarization condition was considered in the spectrum simulation.

The transmission spectrum in Fig. 4a shows resonances separated by the FSR, inversely proportional to the microring length, and characterized by the FWHM and the ER. Higher order modes are observed which have different FSRs, FWHMs and ERs. The presence of higher order modes is due to the guide width, which is relatively large with respect to the operating wavelength range. Analyzing the fundamental mode centered at $\lambda_{P 1}=1.56027 \mu \mathrm{m}$, one can see that FSR, FWHM and $E R$ are $4.13 \mathrm{~nm}, 0.04 \mathrm{~nm}$ and $1.8 \mathrm{~dB}$, respectively. The $Q$ factor of this resonance is $3.9 \times 10^{4}$. The E-field profiles in each one of the two first resonance modes can be obtained with continuous-wave (CW) pumping at the desired wavelength. To this end, two simulations with the Gaussian mode profile centered at the estimated resonances $P_{1}$ and $P_{2}$ were performed, as shown in the inset spectrum of fig. $4 \mathrm{a}$. The electric field profile for these two resonant cases are illustrated in fig. $4 b$.

The spectral response of this device can be considered as a powerful tool in sensors implementations. Selectively changing the refractive index at top of this resonant structure can affect the resonances, representing an interesting feature to be explored in sensing applications.

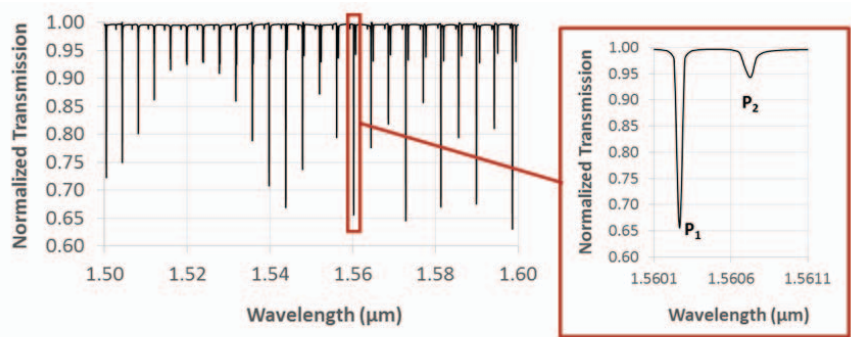

(a)
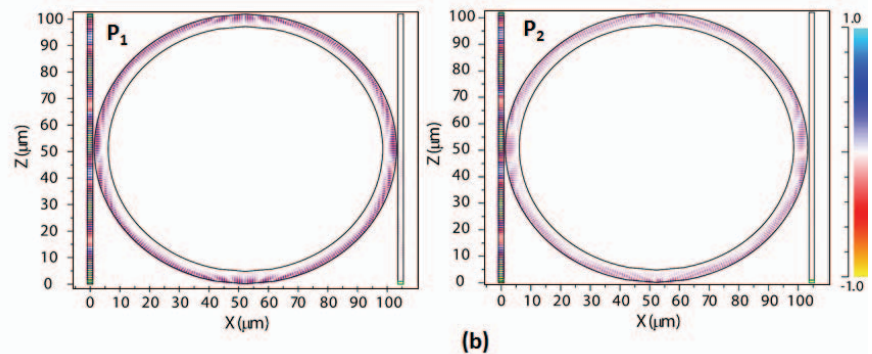

Figure 4: 2-D FDTD simulation results: (a) Transmission spectrum of silicon nitride sensor based on microring resonator. The inset spectrum shows the resonances used to CW-pump. (b) The $\mathrm{E}_{\mathrm{y}}$-field distribution in each one of the resonances $\left(\mathrm{P}_{1}\right.$ and $\left.\mathrm{P}_{2}\right)$ around $1.56 \mu \mathrm{m}$.

To proof this concept, the 2D-FDTD simulation of the sensor was re-performed considering an increase in the background refractive index of $4 \times 10^{-4}$. Fig. 5 illustrates the resonance shift caused by this change in the refractive index. One can see that this shift was $0.03 \mathrm{~nm}$ showing that these devices allow sensitivity about $70 \mathrm{~nm} / \mathrm{RIU}$, where RIU is the refractive index unit. The modification of the refractive index can be physically implemented by another lithography step, where a window is opened on top of the MR, exposing it to a modulation refractive index substance by employing a surface functionalization agent e.g. glutaraldehyde.

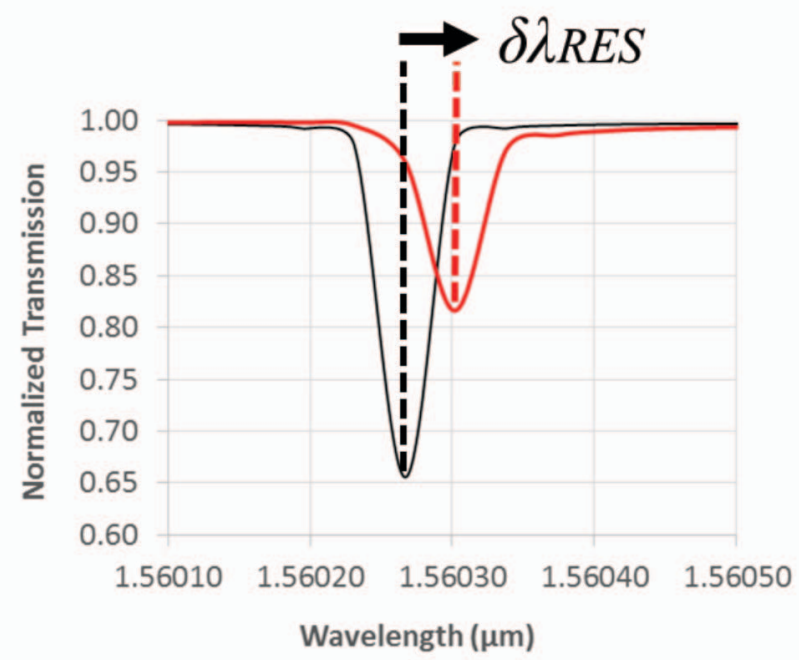

Figure 5: 2D-FDTD Transmission spectrum of silicon nitride sensor based on microring resonator before (black line) and after (red line) a changing in the background refractive index. 


\section{EXPERIMENTAL PROCEDURES}

This section is devoted to present the experimental details of the microresonator fabrication, including DUV lithography and waveguides characterization. Starting by a silicon wafer (2inch diameter, n-type single crystal Si (100) wafers, with resistivity of $1-10 \Omega \mathrm{cm})$, a buffer layer of $\mathrm{SiO}_{2}(3.5 \mu \mathrm{m}$ thick) is deposited, followed by a $\mathrm{Si}_{3} \mathrm{~N}_{4}$ (300 $\mathrm{nm}$ thick), which acts as waveguide.

The wafers were cleaned by using the RCA sequence. In this work it was employed the APCVD technique for the deposition of $3.5 \mu \mathrm{m}$ of a buffer $\mathrm{SiO}_{2}$ layer. The equipment is a reactor from Tempress Systems Inc. The film characterization was performed by employing ellipsometry technique. The equipment used was an UVisel2 from Horiba Scientific. The wafer was measured in 5 regions. The average thickness and refractive index was $3660 \mathrm{~nm} \pm 3.6 \%$ and 1.434 $\pm 0.5 \%$, respectively.

Following the sequence, silicon nitride was deposited by LPCVD. The film characterization was performed by ellipsometry technique. The wafer was also measured in 5 regions, resulting an average $\mathrm{Si}_{3} \mathrm{~N}_{4}$ thickness and refractive index of $290 \mathrm{~nm} \pm 1.0 \%$ and $2.112 \pm 0.5 \%$, respectively.

The DUV optical lithography was performed using a MB6 exposure tool with a special filter for $248 \mathrm{~nm}$ wavelength exposure (HBO 1000W/D, mercury short arc lamp from OSRAM). The process conditions as well as its sequence are summarized in Table I.

Nitride thin layer was etched by using an ICP tool (Roth \& Rau Microsys 400 ICP-RIE system). It has an 8-inch electrode and the control of the etching is made by interferometric monitoring. SF6-based plasma etching was carried out, with the following parameters: RF power of 150 $W\left(0.47 \mathrm{~W}^{\mathrm{cm}} \mathrm{cm}^{2}, 13.56 \mathrm{MHz}\right), 20 \mathrm{mTorr}, 50 \mathrm{sccm}$, during $480 \mathrm{~s}$, resulting in an etching rate of $36 \mathrm{~nm} / \mathrm{min}$.

TABLE I. PROCESS CONDITIONS AND SEQUENCE OF THE DUV LITHOGRAPHY.

\begin{tabular}{|l|l|}
\hline \multicolumn{1}{|c|}{ STEP } & \multicolumn{1}{c|}{ CONDITIONS } \\
\hline RESIST & $\begin{array}{l}\text { UV 210-06 Positive DUV resist } \\
\text { (DOW Chemical) }\end{array}$ \\
\hline PRE-BAKING & $140^{\circ} \mathrm{C}, 5 \mathrm{~min}$ \\
\hline WAVELENGTH & $190 \mathrm{~nm}$ (DUV lithography) \\
\hline EXPOSURE TOOL & Suss-Microtec MA-6, \\
\hline UV POWER & $\begin{array}{l}1.25 \mathrm{~mW} / \mathrm{cm}^{2} \\
40 \mathrm{~mJ} \rightarrow(40 / 1.25)=32 \mathrm{sec}\end{array}$ \\
DOSE & $\begin{array}{l}30 \mathrm{sec} \text { at } 900 \mathrm{rpm}+\text { aceleration of } 500 \\
\mathrm{rpm} / \mathrm{sec} \text { during } 7 \mathrm{sec} .\end{array}$ \\
\hline SPIN COATING & $120^{\circ} \mathrm{C}, 1 \mathrm{~min}$ \\
\hline POST-BAKING & $\mathrm{MF-CD}-26,30 \mathrm{sec} ., 25^{\circ} \mathrm{C}$ \\
\hline DEVELOPMENT & DI water. \\
\hline SAMPLE RINSE & \multicolumn{2}{|l}{} \\
\hline
\end{tabular}

Fig. 6 shows a SEM micrograph of the microring resonator fabricated by DUV lithography. The resulting dimensions match relatively well with respect to the device's design.

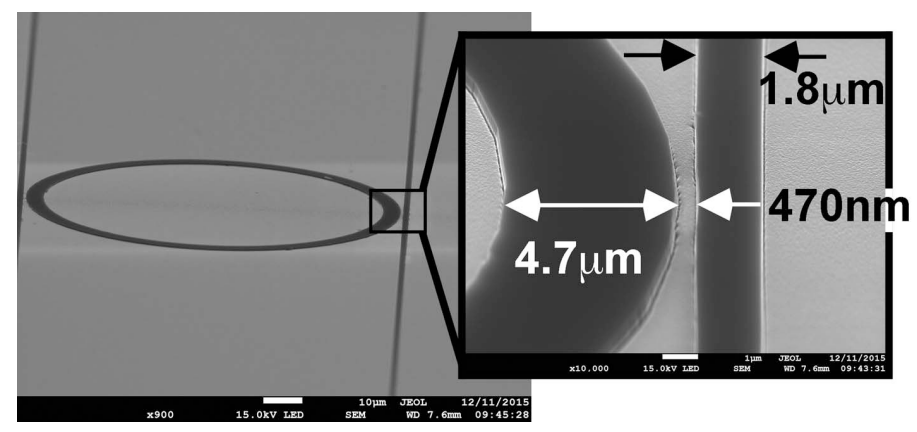

Figure 6: SEM micrograph of the microring resonator, showing the key dimensions of the device.

\section{CONCLUSIONS}

The present work describes the design and fabrication of a microring resonator based on silicon nitride waveguide on top of a silicon dioxide buffer layer.

Simulation results show a $F S R=4.13 \mathrm{~nm}, F W H M=0.04$ $\mathrm{nm}$ and $E R=1.8 \mathrm{~dB}$. The device was fabricated by employing DUV lithography and ICP-RIE plasma etching. From this fabrication scheme, an evanescent coupling gap of $470 \mathrm{~nm}$ was successfully open, which is with good agreement with the employed lithographic tool. The fabricated device has potential application to be used as label-free biochemical sensor, acting as a transducer with receptor molecules immobilized on its surface.

\section{ACKNOWLEDGMENT}

The authors would like to thank the Laboratory of Research on Devices (LPD), Center for Semiconductor Components (CCS) and Laboratório Multiusuários (LAMULT) at University of Campinas. This work was supported by agencies CNPq, (grant \# 203646/2014-2). This work is part of the INCT-NAMITEC, sponsored by CNPq (grant \# 573738/2008-4) and FAPESP (grant \# 2008/57862-6). This work is part of the INCT-FOTONICOM, sponsored by CNPq (grant \# 57857/2008-2) and FAPESP (grant \# 2014/04748-2).

\section{REFERENCES}

[1] G.Robinson, Sens. Actuators B, vol. 29, pp. 31-36 (1995).

[2] S. Blair and Y. Chen, Appl. Opt., vol. 40, pp. 570- 582, (2001).

[3] J. L. Nadeau et al, Proc. SPIE, vol. 4629, pp. 172-180 (2002).

[4] B. Di Bartolo and O. Forte, Frontiers of Optical Spectroscopy. Amsterdam: Verlag, pp. 337-357 (2005).

[5] W. Bogaerts et al, J. Sel. Top. Quantum Electron. 12(6), pp. 1394 1401 (2006).

[6] J. Heebner et al, Optical Microresonators: Theory, Fabrication and Applications, Springer (2008).

[7] O. Schwelb, J. Lightwave Technol. 22(5), pp. 1380-1394 (2004).

[8] N. Yebo, P. Lommens, Z. Hens, and R. Baets, Opt. Express 18(11), pp. $11859-11866$ (2010).

[9] N. Yebo et al, IEEE Photon. Technol. Lett. 21(14), pp. 960-962 (2009).

[10] A. F. Collings and F. Caruso, Rep. Prog. Phys., vol. 60, pp. 1397-1445 (1997).

[11] S. Feng et al.: Laser Photonics Rev. 6, No. 2 pp. 145-177 (2012).

[12] Wim Bogaerts et al, Laser Photonics Rev. 6, No. 1, pp. 47-73 (2012). 